\title{
A MOMENT PROBLEM ON JORDAN DOMAINS
}

\author{
MAKOTO SAKAI
}

ABSTRACT. Let $D_{1}, D_{2}$ be Jordan domains on the complex $z$-plane such that $\int_{D_{1}} z^{n} d m=\int_{D_{2}} z^{n} d m$ for every nonnegative integer $n$. Here $m$ denotes two-dimensional Lebesgue measure. Does it follow that $D_{1}=D_{2}$ ? This moment problem on Jordan domains was posed by H. S. Shapiro [2, p. 193, Problem 1]. In this paper we construct a counterexample and study conditions on $D_{1}$ and $D_{2}$ which imply that the above equality does not hold for some $n$.

1. A counterexample. We first construct a counterexample. Let $R_{1}=\{z|3<| z+1 \mid<\sqrt{10}\}, \quad R_{2}=\{z|3<| z-1 \mid<\sqrt{10}\}, \quad E=R_{1} \cap R_{2}$ $\cap\{z \mid \operatorname{Im} z>0\}$ and $F=\left[\left\{\Delta_{3}(-1) \cap \Delta_{3}(1)\right\}-\left\{\Delta_{1}(-1) \cup \Delta_{1}(1)\right\}\right] \cap\{z \mid \operatorname{Im} z$ $>0\}$, where $\Delta_{r}(c)$ denotes the open disc with radius $r$ and center at $c$. Set $D_{1}=\left\{R_{1} \cup \Delta_{1}(1) \cup \bar{F}\right\}^{\circ}-\bar{E}$ and $D_{2}=\left\{R_{2} \cup \Delta_{1}(-1) \cup \bar{F}\right\}^{\circ}-\bar{E}$, where $\bar{F}$ denotes the closure of $F$ and $A^{\circ}$ denotes the interior of $A$ (Figure $1, D_{2}$ is the reflection of $D_{1}$ in the imaginary axis). Since

$$
\int_{R_{1} \cup \Delta_{1}(1)} z^{n} \cdot d m=\pi\left\{(-1)^{n}+1^{n}\right\}=\int_{R_{2} \cup \Delta_{1}(\dot{-}-1)} z^{n} d m,
$$

two distinct Jordan domains $D_{1}$ and $D_{2}$ satisfy

$$
\int_{D_{1}} z^{n} d m=\int_{D_{2}} z^{n} d m
$$

for every nonnegative integer $n$.

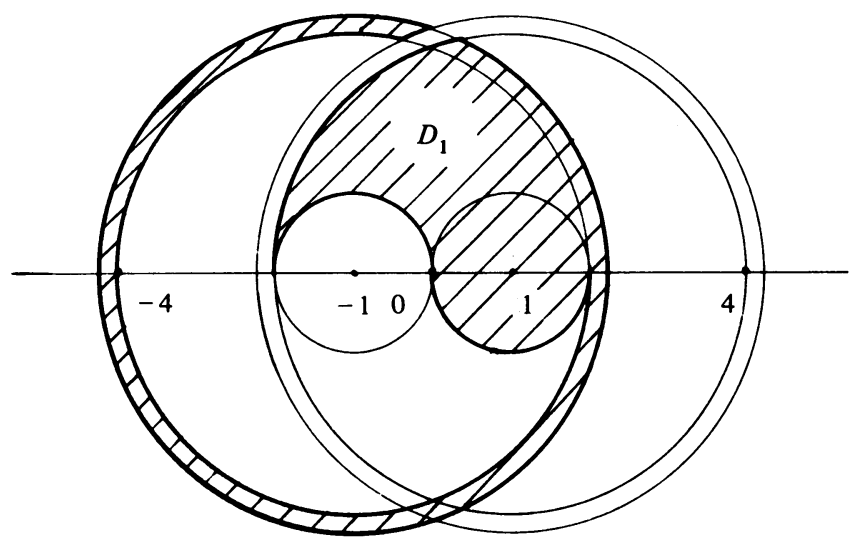

FIGURE 1

Received by the editors September 9, 1977 and, in revised form, October 6, 1977. AMS (MOS) subject classifications (1970). Primary 30A80; Secondary 30A82.

Key words and phrases. Jordan domains, moment problems, polynomial approximation.

c) American Mathematical Society 1978 


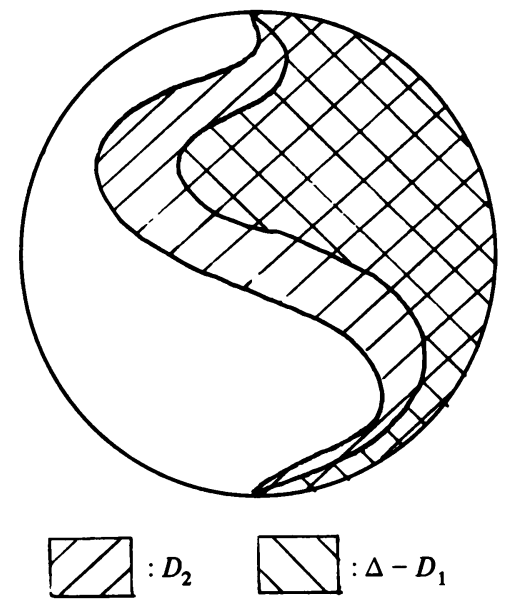

FIGURE 2

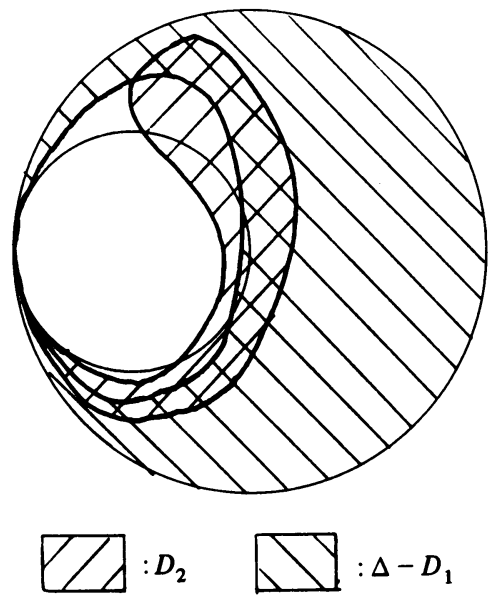

Figure 3

REMARK. By deforming $F$ in the above counterexample we can construct another counterexample with $D_{1}$ and $D_{2}$ which are not congruent.

2. Conditions. We next summarize the known results.

Proposition 1. Let $D_{1}, D_{2}$ be Jordan domains satisfying one of the following conditions:

(i) $D_{1}$ and $D_{2}$ are distinct and there is an analytic $L^{1}$ function $\phi$ on a Jordan domain containing $D_{1} \cup D_{2}$ such that $\operatorname{Re} \phi(z)>0$ on $D_{1}-D_{2}$ and $\operatorname{Re} \phi(z)<$ 0 on $D_{2}-D_{1}$.

(ii) $\bar{D}_{1}$ and $\bar{D}_{2}$ are disjoint or intersect in just one point.

(iii) We denote by $\gamma$ the boundary of the unbounded complementary component of $\bar{D}_{1} \cup \bar{D}_{2}$ and denote by $D_{i}^{e}$ the exterior of $D_{i}$. The restriction of $\gamma$ to $D_{1}^{e} \cup D_{2}^{e}$ is not analytic.

Then

$$
\int_{D_{1}} z^{n} d m \neq \int_{D_{2}} z^{n} d m
$$

for some nonnegative integer $n$.

Condition (i) is very useful. Condition (ii) was given by H. S. Shapiro [2] (see also [1]) and condition (iii) was given implicitly by $\mathrm{D}$. Aharonov and $\mathrm{H}$. S. Shapiro [1, Lemmas 2.2 and 6.1] (see also P. J. Davis [4, p. 21]).

Finally we give our new condition. To do so, we recall the following proposition proved in [6].

Proposition A. Let $D$ be a domain containing the origin 0 . Let $\nu$ be an $L^{1}$ function on $\mathrm{C}$ such that $\nu(z) \geqslant k$ a.e. on $D$ for a positive number $k$ and $\nu(z)=0$ a.e. on the complement of $D$. If $f^{\prime}(0)=\int_{D} f^{\prime} \nu d m / \int \nu d m$ for every analytic function $f$ on $D$ such that $\int_{D}\left|f^{\prime}\right|^{2} v d m<\infty$, then $D \subset \Delta_{r}(0)$, where $r=\left(\int \nu d m / k \pi\right)^{1 / 2}$. The equality $\sup _{z \in D}|z|=r$ holds if and only if $\nu(z)=k$ a.e. on $D$ and $D=\Delta_{r}(0)-E$, where $E$ is a relatively closed subset of $\Delta_{r}(0)$ 
such that $E \cap K$ is removable with respect to analytic functions with finite Dirichlet integrals for every compact subset $K$ of $\Delta_{r}(0)$.

By using this proposition we have

Proposition 2. Let $D_{1}, D_{2}$ be two distinct Jordan domains. Suppose there is an open disc $\Delta=\Delta_{r}(c)$ having the following properties:

(i) $D_{1} \cup D_{2} \subset \Delta$.

(ii) $\chi_{D_{2} \cup\left(\Delta-D_{1}\right)}=\chi_{\Omega}$ a.e. on $\mathbf{C}$ for a simply connected domain $\Omega$ such that

(a) $c \in \Omega$,

(b) every analytic $L^{2}$ function on $\Omega$ can be approximated arbitrarily closely in the $L^{2}$ norm by a sequence of polynomials, where $\chi_{\Omega}$ denotes the characteristic function of $\Omega$.

Then

$$
\int_{D_{1}} z^{n} d m \neq \int_{D_{2}} z^{n} d m
$$

for some nonnegative integer $n$.

PROOF. Assume that $\int_{D_{1}} z^{n} d m=\int_{D_{2}} z^{n} d m$ for every $n$ and set $\nu(z)=$ $\chi_{\Omega}(z)+\chi_{D_{2}-D_{1}}(z)$. Then

$$
\begin{aligned}
\int_{\Omega} z^{n} \nu(z) d m & =\int_{D_{2} \cup\left(\Delta-D_{1}\right)} z^{n} d m+\int_{D_{2} \cap\left(\Delta-D_{1}\right)} z^{n} d m \\
& =\int_{\Delta} z^{n} d m+\int_{D_{2}} z^{n} d m-\int_{D_{1}} z^{n} d m=c^{n} m(\Delta) .
\end{aligned}
$$

Since every analytic $L^{2}$ function on $\Omega$ can be approximated by polynomials, we have

$$
f(c)=\int_{\Omega} f \nu d m / \int \nu d m
$$

for every analytic $L^{2}$ function on $\Omega$. From the definition of $\nu(z)$, it follows that $\nu(z) \geqslant 1$ a.e. on $\Omega, \nu(z)=0$ a.e. on the complement of $\Omega, \int \nu d m=\pi r^{2}$ and $\sup _{z \in \Omega}|z-c|=r$. Hence, by Proposition $A$, we have $\chi_{D_{2}-D_{1}}(z)=0$ a.e. on C. Since $D_{1}$ and $D_{2}$ are Jordan domains satisfying $m\left(D_{1}\right)=m\left(D_{2}\right)$, we have $D_{1}=D_{2}$. This is a contradiction.

Proposition 2 is applicable to the case treated in Problem 4 of [2] (Figure 2, cf. Remark). In this case $\Omega$ is a Jordan domain, and so $\Omega$ has an approximation property mentioned in (b) of Proposition 2. Carathéodory domains also have this property; $\Omega$ is called a Carathéodory domain if its boundary coincides with the boundary of the unbounded complementary component of its closure. There are non-Carathéodory domains having this property (see S. N. Mergelyan [5] and J. E. Brennan [3]). Proposition 2 is also applicable to these cases (Figure 3).

REMARK. If $\Omega$ is a Carathéodory domain such that $\Omega^{e}$ is connected, for example, we can omit (a) of Proposition 2. In fact, for every $\zeta \in \Omega^{e}$, $1 /(z-\zeta)$ can be approximated uniformly on $\bar{\Omega} \cup\{c\}$ by polynomials. 
Hence, from (1), we have

$$
\hat{\nu}(\zeta)=m(\Delta) /(c-\zeta)
$$

where $\hat{\nu}$ denotes the Cauchy transform of $\nu$. Since $\hat{\nu}$ is continuous, it follows that $c \in \Omega$.

\section{REFERENCES}

1. D. Aharonov and H. S. Shapiro, Domains on which analytic functions satisfy quadrature identities, J. Analyse Math. 30 (1976), 39-73.

2. O. B. Bekken, B. K. Oksendal and A. Stray (Editors), Spaces of analytic functions, Lecture Notes in Math., vol. 512, Springer-Verlag, Berlin, 1976.

3. J. E. Brennan, Approximation in the mean by polynomials on non-Caratheodory domains, Ark. Mat. 15 (1977), 117-168.

4. P. J. Davis, The Schwartz function and its applications, Carus Math. Monographs, no. 17, Math. Assoc. Amer., 1974.

5. S. N. Mergelyan, On the completeness of systems of analytic functions, Amer. Math. Soc. Transl. (2) 19 (1962), 109-166. MR 24\#A1410.

6. M. Sakai, Analytic functions with finite Dirichlet integrals on Riemann surfaces, Acta Math. (to appear).

Department of Mathematics, faculty of Science, Hiroshma University, Hiroshima, JAPAN 